\title{
Interesting theoretical mechanics design and production of teaching aids
}

\section{Cen Sun}

\author{
School of Heat energy Power and Mechanical Engineering, NCEPU, Baoding Hebei 071003 \\ suncener@163.com
}

Keywords: Theoretical mechanics, teaching aids, Interesting demonstration.

\begin{abstract}
Teaching aids plays a very important role in the classroom teaching. Interesting mechanical demonstration combined teaching is designed in this paper is the two Teaching aid to mechanics principle as the foundation and cleverly integrated into the fun. With the boat shaped Teaching aid demonstration of Celtic stone phenomenon; human form Teaching aid demonstration of rigid body equilibrium conversion, unstable equilibrium and met with the balance between. The production of simple, convenient demonstration teaching aid, and unique shape, appearance, and make the teaching more vivid, full of fun, convenient for teachers and students, has great practical value.
\end{abstract}

\section{Introduction}

Theoretical mechanics teaching and learning is boring and theoretical. Students can't see the experimental phenomena caused by stress directly which is hard for teaching and learning. Teaching equipment is often with large size and hard to handle. It is not easy to carry for class presentation. At the same time, the phenomena is often abstract and lack of vividness. The teaching aids proposed in the paper is simplified and easy to carry which can be presented in class overcoming the disadvantages of traditional teaching aids. The new teaching aids has a beautiful shape and vivid presentation which can increasing the fun of teach.

\section{Research status}

Theoretical mechanics teaching in China has entered a mature stage. Each school has a good level of theoretical mechanics teaching. In order to provide convenience for teachers, the traditional teaching aids are often imitated according the textbooks. Some teaching aids even sought to make each parts consistent with the models in the textbooks. Granted, it does bring convenience for teachers, however, it cannot increase the fun of learning. Moreover, some aids are limited to laboratories and seldom used in the classrooms. Compared with flash demonstration, the teaching aids proposed in the paper reveal the essential features of the model of theoretical mechanics with outstanding interest. The experiments can be finished in few minutes.

\section{Ship type aid}

The aid is a ship type design in this article. It can be used to demonstrate and explore the kaierteshi phenomenon. The so called Celt Stone phenomenon is that rotation of rigid body in a specific direction contributes to dramatic swings changing the direction of rotation of rigid body. In the course of theoretical mechanics teaching content of friction, Celt Stone phenomenon can be a very interesting issue. Presentation and analysis can be executed through the teaching aid which can greatly increase the fun of learning.

\subsection{Structure introduction}

The aid consists of ship-type block and sail. The block is an ellipsoid which is left - right asymmetric. Sail is a single board which is made up of a vertical pole, a counterweight bar and canvas. 
The pole and bar are hinged. Vertical pole is light and hollow tube while the bar is made of solid iron. The pole and bar are stitched in canvas. The structure of the teaching aid is shown in Figure 1.

The ship bottom is a smooth surface which is a symmetrical smooth elliptic surface. The top surface is an elliptic plane. Angle plate attaches to the upper surface of the ship form, and the center point coincides with the short axis of the ellipse plane. Counterweight is mounted on the boat's upper surface. Its rotating shaft is perpendicular to the upper surface of the ship and the shaft is through the center point $\mathrm{pf}$ short axis of the ellipse plane. The structure can adjust the angle of the counterweight. Through adjusting the angle, it can make the boat center and its center of gravity to have a larger deviation in the direction of the long axis of the body to control the rotation of the ship. There are three hitting points on the upper surface. Point A and B are located near both ends of the long axis of the ellipse plane while point $\mathrm{C}$ is on the short axis.

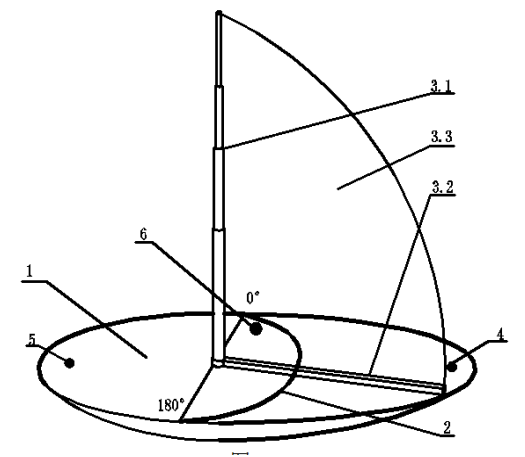

Figure 1

1. body 2. angle scale 3. Counterweight 3.1 vertical pole 3.2 horizontal bar 3.3 canvas 4 . Point A 5 . Point B 6. Point C

\subsection{Demonstration}

By adjusting the vertical pole to change the angle of counterweight, the angle of the counterweight can be ensure according to the angle scale.

The adjustment angle of the counterweight is about 120 degree. Keep the aid still and kick the aid at point $\mathrm{A}$. It can be found that the aid starts to swing around the horizontal axis, and then change to rotate clockwise around a vertical axis. The phenomena of kicking point $\mathrm{B}$ is similar to kick point $\mathrm{A}$. kick the aid at point $\mathrm{C}$ and then it can be found that the aid starts to swing around the horizontal axis, and then change to rotate anticlockwise around a vertical axis. Different kicking position will lead to different rotation direction.

Adjust the angle of counterweight to 120 degree and rotate the aid in the direction of angle decreasing. It can be found that the speed of aid slows down and the swing speed accelerates. Finally, the aid rotates backward and stops at the end. While rotate the aid in the direction of angle increasing, it can be found that the speed of rotation slows down and stops at the end. Different rotation direction has different phenomenon.

Adjust the angle of counterweight to 90 degree and kick point $\mathrm{A}$, point $\mathrm{B}$ and point $\mathrm{C}$ respectively. The aid only has horizontal swing. Toggle the aid in the direction of clockwise and counterclockwise, it can be observed that the aid rotates more and more slowly until it stops. According to the results above, the angle of sail has a direct influence on the rotation of aid.

On the premise of pure rolling, the three phenomena can analyze the swing stability of non-axisymmetric rotating rigid on rough plane. Wobble caused by the friction torque can be obtained. In the course about friction during theoretical mechanics teaching, the phenomenon produced by teaching aids can be regarded as a matter of theoretical mechanics which can be presented and analyzed and increase the fun of learning.

\section{Human type aid}

The human type proposed in the paper is for the study on equilibrium of rigid body type which can be used to present the balance and imbalance of rigid body. It has a good appearance and ornamental. 


\subsection{Structure introduction}

The aid consists of $\mathrm{H}$ platform 5, Humanoid torso 1, arms 2, counterweight 3, 4 and balance bar 6 . Platform 5 is smooth on which there is humanoid torso 1 . The connection of humanoid torso and platform is a two-point contact, the feet and the upper surface. The feet are designed for circular shape or tip shape. In order to facilitate the adjustment of balance, the gravity center of the platform is arranged between the two feet and the humanoid torso legs are designed for the four inverted pyramid structure which are connected with the platform.

There are two arms on both sides of the humanoid torso using bolts for connection. It can adjust the angle between torso and arms. At the end of the arms, a balanced bar is placed. Mortise joints are used between arms and balanced bar which makes the aid more vivid. On both sides of the balanced bar, there are a counterweight and a prolonged pole. The prolonged pole is a screw pile. Threaded connection is used between prolonged pole and counterweight. And the counterweight can be fixed at any position of the prolonged pole. Increase the weight of the counterweights on both sides and make them swing. After a few moments, the humanoid torso will be back to the original equilibrium position. Using minimal disturbance to present the phenomenon, it will make the experiment more vivid and identify the type of balance.

Moreover, in order to get the aid demonstration effect close to the equilibrium phenomenon, the human torso and arms are made of light and sturdy material. Balanced bar and prolonged pole use sturdy metal material. Counterweight uses metal material with high density. The utility has simple structure, beautiful appearance and low production cost. Also it is easy for presentation and have high reliability.

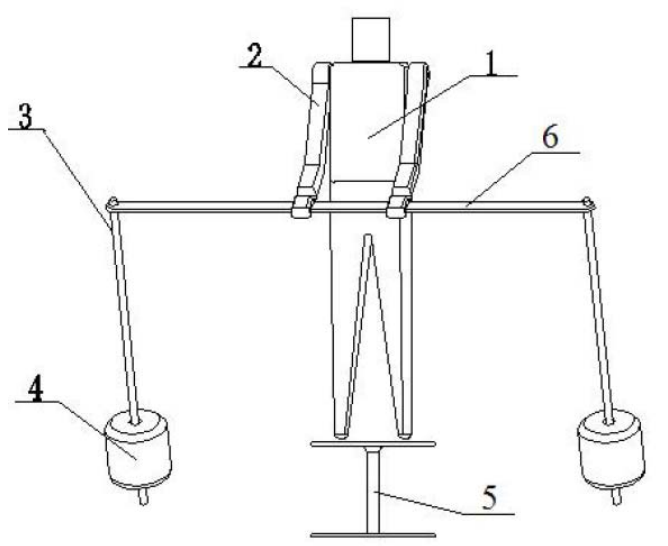

Figure 2

1. humanoid torso 2. arms 3. prolonged pole 4. counterweight 5. H platform 6. balanced bar

\subsection{Demonstration}

As is shown in Figure 2, bolt connection is used between arms and torso. Fixed the counterweight on prolonged pole and make it lower than the upper surface of $\mathrm{H}$ platform. With minimal disturbance on the back, the aid begins to swing and keep balance in the end. At this moment, the aid is in a balanced state.

As is shown in Figure 3, the balanced bar, the prolonged pole and the counterweight are removed. Adjust the angle between arms and humanoid torso and make the aid stands on the platform. With minimal disturbance on the back, the aid will fall down and cannot keep balance. At this moment, the aid is in an unbalanced state. 


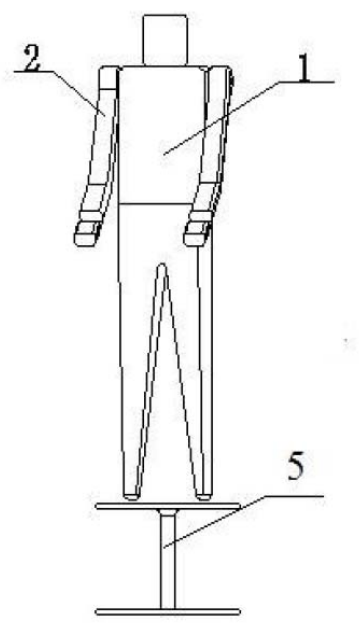

Figure 3

1. human torso 2. arms 3. H platform

Through the programs above, the types of balance can be easily presented. It is vivid and easy to understand. The aid has a small volume and is easy for presentation. The utility has simple structure, beautiful appearance and low production cost. Also it is easy for presentation and have high reliability.

\section{Summary}

1. The ship type aid in this paper ingeniously equips the counterweight as a sail in the application which makes the aid more ornamental and novel. By changing the position of the sail, the aid can be used to study the effect of Celt Stone phenomenon.

2. Recently, the aid to study the balance problem is main designed according to equilibrium of two forces. It mainly consists of fine lines, weights and pulleys which is boring and simple. Balance type is divided into three, namely the stable balance, the equilibrium and the indifferent equilibrium which make up for the vacancy of teaching aids about different balance types.

\section{References}

[1] Liu Y S. On Dynamics of Celtic Stone [J]. Journal of Mechanics in Engineering. 1991(04): p: 52-54. (in Chinese)

[2] Theoretical Mechanics Department of Harbin Institute of Technology. Theoretical Mechanics. High Education Press .2009:p45-46. (in Chinese) 